\title{
Nonword Facilitation in a Lexical Decision Task
}

\author{
Sachiko Kinoshita, Marcus Taft, and John E. Taplin \\ University of New South Wales, Kensington, Australia
}

\begin{abstract}
It has been reported in previous experiments that in a lexical decision task, sentence contexts facilitate decisions for nonword targets relative to a neutral context condition (e.g., Schuberth \& Eimas, 1977). The present three experiments investigated possible explanations for this nonword facilitation effect, using moderately predictable sentence contexts. Results provided support for the view that nonword facilitation stems from an overestimate of the baseline decision latency when a neutral context condition and sentence context conditions are intermixed. Specifically, it was suggested that context affects a post-access decision stage during which sentential meaningfulness is used as a basis for making wordnonword discriminations and that nonword facilitation stems from an extra processing stage required for targets following a neutral context. Implications of this interpretation for the effects of sentence context on the recognition of words are discussed.
\end{abstract}

Recent studies of word recognition have emphasized the role played by semantic context (e.g., Becker, 1980; Schuberth \& Eimas, 1977; Stanovich \& West, 1981). In particular, it has been suggested that the processes involved in recognizing a word may be fundamentally different for a word presented in isolation and for a word presented in a sentence context. These studies have typically presented words following a semantically congruous sentence context (e.g., The boy swam under the BRIDGE) or an incongruous sentence context (e.g., The cook baked the BRIDGE) and have found that the response to a target word is faster in the former condition. Many investigators have discussed this effect in terms of facilitation and inhibition effects, where facilitation refers to faster response latencies to words following a congruous context relative to a semantically neutral context,

Portions of this article were presented at the Ninth Experimental Psychology Conference held at Brisbane, Australia, in May 1982. The authors wish to thank Sally Andrews, Ken Forster, and Sue Wayland for their helpful discussion prior to the preparation of the manuscript. Thanks are also due to the reviewers who provided comments on an earlier version of the paper, namely, Curtis Becker, Ira Fischler, Kirk Smith, an anonymous reviewer, and the editor, Henry Roediger.

Requests for reprints should be sent to Sachiko Kinoshita, Department of Psychology, University of New South Wales, P. O. Box 1, Kensington, New South Wales, Australia, 2033. and inhibition refers to slower latencies for words following an incongruous context.

In making these comparisons, it has been assumed that the neutral context condition serves as a baseline condition; that is, it is equivalent to a sentence context in all respects (for example, in terms of its alerting property, the processing capacity required to read the context, etc.) except that a neutral context does not constrain the likely target words semantically. Various types of neutral contexts have been used in previous investigations, for example, a row of $X$ s (Fischler \& Bloom, 1979), a blank field (Schuberth \& Eimas, 1977), the word the (Stanovich \& West, 1979) a random list of words (Forster, 1981), sentence fragments such as It was the (Stanovich \& West, 1981), and so forth. However, it appears that not all of these neutral context conditions provide an appropriate baseline, because in a lexical decision task, nonword targets have been found to produce faster decision latencies when they are preceded by a sentence context compared with a neutral context (e.g., Schuberth \& Eimas, 1977; West \& Stanovich, 1982). Because nonwords, by definition, are not part of the lexicon and therefore have no reason to be affected by context, a finding of such nonword facilitation casts a doubt on the appropriateness of some of these neutral contexts. The aim of this article is to investigate the locus of this nonword facilitation effect and in turn to 
discuss its implication for the role of sentence contexts in the recognition of words.

At least four different explanations for the nonword facilitation effect have been suggested: they will be referred to as the predictand-match account, semantic-matching account, lexical warm-up hypothesis, and processing-load explanation. Each of these accounts will be described in turn, and we will argue that none of these can adequately address the existing data.

The first of these, the predict-and-match explanation, was proposed by Neely (1976). In his experiment, the semantic context consisted of a single word for which the target word was a primary associate (e.g., dog-CAT, doctor-NURSE), and the neutral context, a row of $X \mathbf{s}$. By using a lexical decision task, he found both a facilitation effect for related target words, an inhibition effect for unrelated target words (i.e., a slower response latency for targets following an unrelated-incongruous context relative to the neutral context), as well as a nonword facilitation effects. Neely suggested that in his experiment, subjects were actively predicting a likely target word from the context and were biased toward responding word if the prediction matched the target and nonword if it did not. As Neely suggested, such a strategy should result in the pattern of data obtained.

Although Neely's (1976) explanation was proposed for the results obtained with single word contexts, there is no reason why it should not be applied to experiments that used sentence contexts. That is, subjects may be able to use the semantic information contained in the sentence context to predict a likely target word. However, one problem with this explanation is that nonword facilitation has been obtained in experiments in which subjects were unlikely to have used the predictive strategy. For example, Schuberth and Eimas (1977) reported finding nonword facilitation in a lexical decision task in which the sentence contexts used had relatively low Cloze probability (all contexts had Cloze probability of less than .70, and averaged .12; cf. Fischler \& Bloom, 1979). It seems unlikely that subjects were able to successfully predict target words in such a situation. Furthermore, Fischler and Bloom (1979, 1980) reported that no corresponding increase in the size of facilitation was found for targets following sentence contexts with greater predictability. Such a finding is at odds with the notion that sentence contexts are used to predict a likely target word.

Another finding that argues against the predict-and-match interpretation of the effects of sentence context is the pattern of interaction between the effects of context and target word frequency. In a lexical decision task involving decisions for words presented in isolation, one of the most important determinants of decision latency is the frequency of the target word (e.g., Forster \& Chambers, 1973; Scarborough, Gerard, \& Cortese, 1977; Whaley, 1978). This word frequency effect (i.e., high-frequency words are responded to faster than low-frequency words) is generally attributed to a process that occurs during lexical access and is interpreted either as reflecting the order in which lexical search takes place (e.g., Forster, 1976) or as indicating a higher level of threshold activation for highfrequency words (e.g., Morton, 1969). However, this effect has been found to be reduced for words following a related word (Becker, 1979; Bednall, cited in Forster, 1976). This suggests the possibility that the target word may have been predicted from the context, rather than being accessed via the bottom-up lexical access process, which is responsible for frequency effects. On the other hand, in studies involving sentence contexts, generally the effects of frequency and context have been found to be additive (e.g., Forster, 1981; Schuberth \& Eimas, 1977; Schuberth, Spoehr, $\&$ Lane, 1981). ${ }^{1}$ The absence of such fre-

\footnotetext{
' Contrary to the finding by other investigators, Stanovich and West (1981; 1983; West \& Stanovich, 1982) have reported finding a reduced effect of word difficulty (where "difficult" words were both longer and of lower frequency) following a congruous sentence context relative to a neutral context. However, the reliability of these findings is open to question. First, Forster (1981, Experiments 4 \& 5), using the same stimuli and task (i.e., a naming task) but a different procedure for presenting the context, failed to replicate this interaction. Second, Stanovich and West obtained the interaction by always using the same context and target stimuli. Third, they rarely report the results of items analysis, thus suggesting the possibility that the interaction may be due to some, but not all of the items. For those reasons it was considered that this finding of an interaction may be peculiar to the stimuli or procedure used by Stanovich and West.
} 
quency by context interaction may thus in turn suggest that when sentence contexts are used, they are not used to predict the target words.

Neely (1977) in fact later proposed an alternative interpretation in order to account for the data from another experiment where nonword facilitation was obtained in a situation in which subjects were unlikely to have been predicting a particular target word. In this experiment using category names as contexts, equal facilitation was obtained for targets that were high-typical members of a category (e.g., bird-ROBIN) and low-typical members of a category (e.g., bird-PARROT). Such absence of a typicality effect is difficult to reconcile with the view that subjects were actively predicting a target word from the context. Neely therefore proposed the following explanation.

According to this view, referred to as the semantic-matching explanation, the subject does not generate a particular word as a likely target, but instead generates a set of semantic features expected from the category context and responds on the basis of the similarity between the semantic features of the expected word and the semantic features activated by the actual target string. If the semantic features of the expected word and that of the actual target are similar, the subject would respond word; if they are dissimilar, the subject would have the tendency to respond nonword. Such a response strategy would result both in word and nonword facilitation effects, and in a word inhibition effect, as were obtained in the experiment. Neely further argued that the absence of a typicality effect is also consistent with this semantic-matching account on the basis of a finding by Rosch (1975). Rosch reported a faster decision latency for high-typical targets than for low-typical targets following a category name when the required decision was whether or not the two target words were physically identical (e.g., bird-ROBIN-ROBIN), but equal decision latencies for high- and low-typical members when the required decision was whether or not the two physically nonidentical targets belonged to the same category (e.g., bird-ROBIN-SPARROW). Neely took this finding to suggest that equal facilitation is obtained for high- and low-typical targets following a category name, whenever the subject is making some sort of a semantic decision.

This semantic-matching explanation is not free of problems either, however. For one, Neely's account of the absence of a typicality effect is inconsistent with other findings: there is evidence that a greater facilitation is obtained for high-typical targets than for lowtypical targets following a category name in a task that clearly requires a semantic decision. For example, Sanford, Garrod, and Boyle (1977) obtained greater facilitation for high-typical targets when the decision required was whether or not the target belonged to the specified category (i.e., decision latency was faster for bird-ROBIN than for bird-PARROT). Thus the absence of a typicality effect cannot be used as unequivocal evidence for the involvement of a semantic-matching strategy.

A second problem with the semanticmatching strategy account is that in the case of experiments involving sentence contexts, it is often difficult to find common semantic features among words that may form a meaningful completion for a given sentence context (cf. Forster, 1981; Kleiman, 1980). To illustrate this point, take the following sentence context: The puppy chewed the. A number of words may meaningfully complete this context, for example, bone, shoe, ball, and so forth. However, it is difficult to find semantic features that are common to these words (apart from the fact that they are things a puppy can chew). Thus short of predicting individual words (a possibility that has been rejected earlier), it seems unlikely that a sentence context may be used to generate semantic features for likely target words.

A third explanation for the nonword facilitation effect may be termed the lexical warmup explanation. According to this account, sentence contexts, because they contain words, may "actively engage the lexical processor prior to the occurrence of a target" (Forster, 1981) and therefore place decisions for targets following a row of $X$ s at a relative disadvantage. A similar explanation suggests that a row of $X s$ is less alerting than linguistic material (e.g., Antos, 1979; Kleiman, 1980). However, these explanations are at odds with West and Stanovich's (1982) finding that nonword responses were slower following a neutral context consisting of the sentence fragment They said it was the in comparison 
with the sentence context condition. If nonword facilitation were simply due to a lack of lexical warm-up or difference in the alerting value, then nonword facilitation should have disappeared when the neutral context contained words instead of a row of $X \mathbf{s}$.

Finally, a fourth or processing-load explanation suggests that the nonword facilitation effect may be attributable to differing demands placed on the central processor when reading sentence and neutral contexts. Kleiman (1980) pointed out that the contexts requiring a greater processing load may slow down responses to subsequent stimuli. He cited as an example the experiment conducted by Schuberth and Eimas (1977), in which the neutral context consisting of spelled-out digits (e.g., THREE SIX FIVE NINE) slowed down lexical decisions to subsequent targets by 70 ms compared with when the same targets were preceded by a neutral context consisting of a blank field. However, this explanation predicts a pattern of nonword facilitation effects that is contrary to the pattern that has actually been obtained: nonword decisions are in fact found to be faster following a sentence context than a neutral context, which should require less processing load.

As none of the available explanations can account for the pattern of previous findings, it would seem necessary to seek an alternative explanation for the nonword facilitation effect. In particular, it is important that the explanation be capable of accounting for the presence of nonword facilitation in some experiments and its absence in others. Of those experiments that specifically reported the nonword data, it is notable that Fischler and Bloom $(1979,1980)$ did not obtain nonword facilitation by using two types of neutral contexts. Fischler and Bloom's procedure differed from other investigators' in that they presented the neutral context condition to a separate group of subjects rather than randomly mixing neutral and sentence context conditions. This difference in the mode of presentation may then be responsible for the difference in the pattern of nonword data reported.

It has been noted in previous experimental tasks that mixed and blocked presentations of different conditions yield different decision latencies for the same items. For example, Glanzer and Ehrenreich (1979) reported that lexical decisions for high frequency words were slower when they were mixed randomly with low and medium frequency words compared with when they were presented in a "pure" list. Kiger and Glass (1981) reported that in an arithmetic verification task, the same items were responded to more slowly when they were mixed with difficult items compared with when they were mixed with easy items. Kiger and Glass interpreted these findings as suggesting that subjects adopt a more conservative criterion when difficult items are added to the same list. It may therefore be possible that a similar shift in decision criterion was responsible for the difference in the pattern on nonword data between Fischler and Bloom's (1979, 1980) experiments using a blocked presentation and others using a mixed presentation.

In order to test this possibility, Experiments 1 and 2 were conducted using the same stimuli, but different modes of presentation. In both experiments, a lexical decision task was used, and the sentence contexts ranged in predictability from fairly low to reasonably high (as indexed by Cloze probability). The neutral context consisted of a row of $X \mathrm{~s}$. Experiment 1 used a mixed presentation of neutral and sentence context conditions, whereas Experiment 2 used a blocked presentation. It was expected that if the mode of presentation is a crucial factor in determining the pattern of nonword facilitation effects, then nonword facilitation should be obtained in Experiment 1, but not in Experiment 2.

In addition to the blocked versus mixed presentation, two other factors were manipulated in the two experiments: these were the frequency of the target word and the predictability of the target word as indexed by Cloze probability. These factors were included primarily to confirm the observations mentioned earlier, namely, that the effects of frequency and sentence context do not interact and that the amount of facilitation is unrelated to the predictability of the target.

\section{Experiment 1}

\section{Method}

Subjects. Twenty-four first-year psychology students at the University of New South Wales participated in the experiment to fulfill their credit time requirements.

Stimuli. There were 72 word targets and 72 nonword 
targets. The word targets were either high or low frequency, and they were assigned to one of the three context conditions; namely, congruous sentence, incongruous sentence, or neutral context. The words ranged from 4 letters to 7 letters in length, the average length being 4.59 letters long. The high-frequency words had 100 or more occurrences per million (average 237.1 per million) and the low-frequency words had 33 or less occurrences per million (average 16.6 per million) according to the Kucera and Francis (1967) word frequency count. The congruous sentence contexts were either selected from Bloom and Fischler's (1980) pool of items or were selected by the experimenters, who used a similar procedure to that of Bloom and Fischler. The Cloze probability of the items ranged between .17 and .78 (average Cloze probability was .48 for both high- and low-frequency words). For the incongruous context condition, the sentence contexts and the word items were re-paired so that the target words would make the sentence as a whole semantically anomalous (e.g., The lady recovered the stolen BEARD). The neutral context consisted of a row of $X$ s approximating a six-word sentence ( $X X X X X X X X X X$ XXXXX XXXXX $\mathrm{XXXX}$ ). Examples of the context types and target words are shown in Table 1.

Each group of high- and low-frequency word targets were divided into three equal sets: $A, B$, and $C$ matched

Table 1

Examples of Targets and Contexts Used and the Number of Trials Per Condition in Experiment I

\begin{tabular}{|c|c|c|c|}
\hline Condition & Context & Target & $\begin{array}{l}\text { Number } \\
\text { of trials }\end{array}$ \\
\hline \multicolumn{4}{|l|}{$\begin{array}{l}\text { High-frequency } \\
\text { word }\end{array}$} \\
\hline Congruous & $\begin{array}{l}\text { He wrote a } \\
\text { letter to his }\end{array}$ & mother & 12 \\
\hline Incongruous & $\begin{array}{l}\text { They could not } \\
\text { survive } \\
\text { without }\end{array}$ & hour & 12 \\
\hline Neutral & $\begin{array}{l}\mathrm{XXX} \times \mathbf{X X X X} \times \mathrm{XX} \\
\mathrm{XXXXX} \\
\mathrm{XXXXX} \\
\mathrm{XXXX}\end{array}$ & water & 12 \\
\hline \multicolumn{4}{|l|}{$\begin{array}{l}\text { Low-frequency } \\
\text { word }\end{array}$} \\
\hline Congruous & $\begin{array}{l}\text { Fred put the } \\
\text { worm on a }\end{array}$ & hook & 12 \\
\hline Incongruous & $\begin{array}{l}\text { Helen reached } \\
\text { up to dust } \\
\text { the }\end{array}$ & bugs & 12 \\
\hline Neutral & 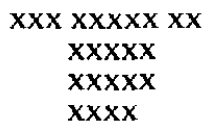 & shelf & 12 \\
\hline $\begin{array}{l}\text { Nonword } \\
\text { Sentence }\end{array}$ & $\begin{array}{l}\text { He drove the } \\
\text { nail into } \\
\text { the }\end{array}$ & gome & 48 \\
\hline Neutral & 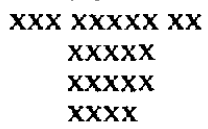 & mosic & 24 \\
\hline
\end{tabular}

as closely as possible on word length, frequency, and Cloze probability. The assignment of these sets to the three context conditions was counterbalanced across the three subgroups of subjects by using a Latin-square design. Thus subjects in Subgroup 1 saw Set $A$ targets in the congruous context condition, Set B targets in the incongruous context condition, and Set $\mathrm{C}$ targets in the neutral context condition; subjects in Subgroup 2 saw Sets $\mathbf{B}, \mathbf{C}$, and $\mathbf{A}$ in the congruous, incongruous, and neutral context conditions, respectively; and subjects in Subgroup 3 saw Sets C, A, and B in the congruous, incongruous, and neutral context conditions, respectively. Therefore any one subject saw a target and a sentence context only once, and across subjects, each target appeared in all three context conditions.

There were also 72 nonword targets in this experiment. As in previous experiments (e.g., Becker, 1980; Fischler \& Bloom, 1979), they were generated by changing one letter of a word target, for example, from the word year, the nonword yeir was generated. The nonword targets were assigned to the three context conditions by using the same counterbalancing procedure as for the word targets, except for the following difference. Because no nonword target appeared in the sentence context that suggested the word from which the nonword was generated (as in The crime rate has gone up this YEIR), there was no distinction between the congruous and incongruous sentence contexts for nonword targets. Examples of nonword targets and contexts are also shown in Table 1.

In addition, there were 12 practice context-target combinations and two filler combinations selected according to the same criteria.

Procedure. Subjects were told at the beginning of the session that the experiment involved deciding whether or not a target item was a word, where a target would be preceded by either a sentence context or a row of Xs. It was also pointed out that when it was a sentence context, the word following it may or may not complete the sentence meaningfully, and it was emphasized that the decision was about whether or not it was a word, not whether or not the sentence as a whole made sense. The importance of reading the sentence was also stressed. The subjects were told to make the lexical decisions as quickly and as accurately as possible.

The stimuli were presented on a video terminal controlled by a Motorola 6809 microprocessor. The items were presented in a green-on-black format, all in capital letters. On each trial, either all words in a sentence frame or a row of $X s$ were presented and they remained on the screen for $3 \mathrm{~s}$. One hundred milliseconds after the offset of the sentence frame or the row of $X \mathrm{~s}$, the target was presented, and remained on the screen for $2 \mathrm{~s}$. The target was presented where the sentence frame or the row of $X$ s ended. Subjects made a word response with the right hand and a nonword response with the left hand. The subjects were tested individually in sound-proof cubicles. Each session lasted approximately $20 \mathrm{~min}$.

\section{Results}

The word and nonword data were analyzed separately. Both decision latency for the correct responses and the number of errors were 
analyzed for each subject's means treating items as a random variable and for each item's means treating subjects as a random variable. An effect was considered to be significant when it was significant both on subjects and items analyses, allowing generalizability to both new subjects and items.

In order to minimize the effect of occasional trials with very long latencies, the mean latencies for word and nonword decisions were calculated for each subject, and upper and lower cutoffs were set $3 S D$ units above and below the means for that subject. Decision latencies above or below these cutoff points were replaced with the cutoff value. A similar procedure was carried out for each item collapsed over subjects.

All contrasts were fully planned, and the Bonferroni decision rule for establishing the significance of each family was used, holding the Type I error rate at .05 .

The word decision latency and error data are displayed in Table 2.

For the word data, the contrasts tested the main effects of two factors, namely, frequency of the target word (high vs. low) and context (congruous, incongruous, and neutral), and the interactions resulting from them. Thus there were five planned contrasts: (1) the main effect of frequency; (2) the main effect of facilitation (i.e., neutral minus congruous); (3) the main effect of inhibition (i.e., incongruous minus neutral); (4) the facilitation by frequency interaction; and (5) the inhibition by frequency interaction. The latency data showed that the main effect of frequency was significant, $F_{1}(1,23)=68.896, M S_{\mathrm{e}}=$ $1,766.423$ and $F_{2}(1,70)=29.577, M S_{\mathrm{e}}=$ 7,628.414. Overall, high-frequency words were responded to $65 \mathrm{~ms}$ faster than low-frequency words. The 58-ms facilitation effect was also significant, $\quad F_{1}(1,23)=36.01, \quad M S_{\mathrm{e}}=$ $2,017.159$ and $F_{2}(1,70)=21.516, M S_{\mathrm{e}}=$ $5,639.939$. These two effects did not interact, $F_{1}(1,23)<1.0$ and $F_{2}(1,70)<1.0$. The $44.5-$ ms inhibition effect was significant, $F_{1}(1$, $23)=7.043, \quad M S_{\mathrm{e}}=4,570.218$ and $F_{2}(1$, $70)=14.114, M S_{\mathrm{e}}=4,984.088$. Although a greater inhibition effect was observed for lowfrequency words than for high-frequency words, this inhibition by frequency interaction did not reach significance on either subjects analysis, $F_{1}(1,23)=1.343, M S_{\mathrm{e}}=1,679.288$,
Table 2

Mean Latencies (in Milliseconds) and Percentage Error Rates (\% E) for Word and Nonword Decisions in Experiment I

\begin{tabular}{lcccccr}
\hline & \multicolumn{6}{c}{ Frequency } \\
\cline { 2 - 7 } Condition & High $\% \mathrm{E}$ & Low $\% \mathrm{E}$ & $\begin{array}{c}\text { Differ- } \\
\text { ence }\end{array}$ & $\% \mathrm{E}$ \\
\hline Word & & & & & & \\
$\quad$ Congruous & 615 & 2.1 & 658 & 1.4 & 43 & -0.7 \\
$\quad$ Incongruous & 693 & 4.5 & 785 & 7.6 & 92 & 3.1 \\
$\quad$ Neutral & 665 & 1.0 & 724 & 2.1 & 59 & 1.1 \\
Nonword & & & & & & \\
$\quad$ Sentence & 827 & 2.7 & & & & \\
$\quad$ Neutral & 860 & 4.5 & & & & \\
\hline
\end{tabular}

Note. Facilitation and inhibition effects for word contrasts were $58(\% \mathrm{E}=-0.2)$ and $44.5(\% \mathrm{E}=4.5)$, respectively. Facilitation effect for nonword contrast was 33 (\% $\mathrm{E}=$ 1.8).

or items analysis, $F_{2}(1,70)=3.475, M S_{\mathrm{e}}=$ $2,806.944$.

The error data generally showed the same trend as the latency data; however, none of the contrasts reached significance except for the inhibition effect, $F_{1}(1,23)=16.264$, $M S_{\mathrm{e}}=0.433 ; F_{2}(1,70)=13.661, M S_{\mathrm{e}}=$ 0.344 . None of the $F$ values for other contrasts reached 1.0 , except for the main effect of frequency, $F_{1}(1,23)=1.716, M S_{\mathrm{e}}=.405$ and $F_{2}(1,70)=1.617, M S_{\mathrm{e}}=0.286$, and the facilitation by frequency interaction, $F_{1}(1$, $23)=2.003, M S_{\mathrm{e}}=0.13$ and $F_{2}(1,70)=$ $1.304, M S_{\mathrm{e}}=0.133$.

The nonword decision latency and error data are also displayed in Table 2. Only one contrast was tested for the nonword data: the effect of context (sentence vs. neutral). Nonword decisions were significantly faster following sentence contexts than neutral contexts, $F_{1}(1,23)=16.3, M S_{\mathrm{e}}=2,247.174$ and $F_{2}(1,71)=12.112, M S_{\mathrm{c}}=5,154.08$. The error data also showed that significantly more errors were made in the neutral context condition than in the sentence context condition, $F_{1}(1,23)=5.375, M S_{\mathrm{e}}=0.285$ and $F_{2}(1$, $71)=6.138, M S_{\mathrm{e}}=0.255$. In addition, in order to test whether the size of facilitation was dependent on the predictability of the target from the sentence context, the Pearson product-moment correlation coefficient was calculated between the size of facilitation $\left(\mathrm{RT}_{\text {neutral }}-\mathrm{RT}_{\text {congruous }}\right)$ and Cloze probability. 
It should be recalled that the design involved a between-subjects counterbalancing of target words across the three context conditions. Consequently, the calculation of the facilitation effect for a given target involved the subtraction of the decision latencies averaged over a subgroup of subjects in the congruous sentence context condition from the decision latencies averaged over another subgroup of subjects in the neutral context condition. Such a method of computing the size of the facilitation effect could be adversely affected by individual differences in reaction time across subjects. In order to overcome this problem, the "super-subject/item" was used as the unit of analysis. ${ }^{2}$ Within each subset (Sets A, B, and C), target words were rank-ordered in terms of Cloze probability. The decision latencies in the congruous sentence context condition were then averaged over the three targets from the different subsets of the same rank to yield a composite congruous decision latency. Similarly, a composite neutral decision latency was calculated by averaging over three targets. The facilitation score computed from these two composite decision latencies were then used to calculate the Pearson product-moment correlation coefficient with average Cloze probability over the three targets. The correlation was nonsignificant, $r=-.04, p>.10, d f=22$.

\section{Experiment 2}

\section{Method}

Subjects. An additional 24 subjects from the same subject population participated in this experiment. None of the subjects had participated in Experiment 1.

Stimuli. The context and target stimuli were identical to those used in Experiment 1.

Procedure. The apparatus, timing characteristics, and the instructions given to the subjects were identical to those in Experiment 1, except the two sentence context conditions and the neutral context condition were blocked and were presented as two subexperiments. Half of the subjects did the sentence context block first, and the other half did the neutral context block first.

\section{Results}

The analysis of Experiment 2 was the same as that of Experiment 1. The mean latency for correct responses and error rates for word decisions are displayed in Table 3.

The latency data showed that the main
Table 3

Mean Latencies (in Milliseconds) and Percentage Error Rates (\% E) for Word and Nonword Decisions in Experiment 2

\begin{tabular}{lrrrrrr}
\hline & \multicolumn{6}{c}{ Frequency } \\
\cline { 2 - 7 } & & & \multicolumn{5}{c}{$\begin{array}{c}\text { Differ- } \\
\text { Condition }\end{array}$} & High & $\% \mathrm{E}$ & Low & $\% \mathrm{E}$ & ence & $\% \mathrm{E}$ \\
\hline Word & & & & & & \\
$\quad$ Congruous & 657 & 1.7 & 694 & 5.6 & 37 & 3.9 \\
Incongruous & 746 & 13.5 & 816 & 13.9 & 70 & 0.4 \\
$\quad$ Neutral & 677 & 3.1 & 722 & 4.9 & 45 & 1.8 \\
Nonword & & & & & & \\
$\quad$ Sentence & 860 & 10.2 & & & & \\
$\quad$ Neutral & 855 & 8.7 & & & & \\
\hline
\end{tabular}

Note. Facilitation and inhibition effects for word contrasts were $24(\% \mathrm{E}=0.4)$ and $81.5(\% \mathrm{E}=9.7)$, respectively. Facilitation effect for nonword contrast was $-5(\% \mathrm{E}=$ $-1.5)$.

effect of frequency was significant, $F_{1}(1,23)=$ $39.283, M S_{\mathrm{e}}=2,375.225$ and $F_{2}(1,70)=$ $11.51, M S_{\mathrm{e}}=13,830.273$. Overall, the highfrequency words were responded to $51 \mathrm{~ms}$ faster than low-frequency words. Decisions were made $24 \mathrm{~ms}$ faster in the congruous sentence context condition in comparison with the neutral context condition. This facilitation effect was significant on the items analysis, $F_{2}(1,70)=6.334, M S_{c}=6,019.948$, but not on the subjects analysis, $F_{1}(1,23)=$ $1.449, M S_{\mathrm{e}}=9,470.101$. The 81.5 -ms inhibition effect, on the other hand, was significant on both, $F_{1}(1,23)=13.242, M S_{\mathrm{e}}=$ $12,019.811$ and $F_{2}(1,70)=25.903, M S_{\mathrm{e}}=$ $7,411.519$. The interaction between the facilitation effect and the effect of frequency was nonsignificant, $F_{1}(1,23)<1.0$ and $F_{2}(1$, 70) < 1.0. As in Experiment 1, a greater inhibition effect was observed for low-frequency words than for high-frequency words, but again this interaction did not reach significance, $F_{1}(1,23)<1.0$ and $F_{2}(1$, $70)=2.12, M S_{\mathrm{e}}=7,411.519$.

The error data generally showed the same trend as the latency data. The effect of frequency reached significance on the items analysis, $F_{2}(1,70)=6.101, M S_{\mathrm{e}}=0.513$, but not on the subjects analysis, $F_{1}(1,23)=$

\footnotetext{
2 We are grateful to Curtis Becker for suggesting this method of analysis.
} 
2.589, $M S_{\mathrm{e}}=0.775$. Again the facilitation effect failed to reach significance, $F_{1}(1,23)<$ 1.0 and $F_{2}(1,70)<1.0$. The inhibition effect was reliable, $F_{1}(1,23)=30.255, M S_{\mathrm{e}}=1.08$ and $F_{2}(1,70)=14.108, M S_{\mathrm{e}}=0.539$. All other contrasts yielded an $F$ value of less than 1.0.

The nonword decision latency and error data are also displayed in Table 3. As opposed to Experiment 1, decisions were faster in the neutral context condition than in the sentence context condition, but this difference was not significant, $F_{1}(1,23)<1.0$ and $F_{2}(1,71)<$ 1.0. Similarly, there were slightly fewer errors made in the neutral context condition, but again this difference was not significant, $F_{1}(1$, 23) $<1.0$ and $F_{2}(1,71)<1.0$.

As in Experiment 1, the Pearson productmoment correlation was calculated between the amount of facilitation and Cloze probability by using the method described earlier. The correlation was again nonsignificant, $r=$ $.34, p>.10, d f=22$.

\section{Discussion}

The results of the two experiments showed that a blocked presentation of neutral and sentence context conditions eliminated the nonword facilitation effect obtained with a mixed presentation. It can also be seen that the pattern of facilitation and inhibition effects for word decision data was different for the two experiments, indicating that the blocked presentation resulted in a smaller facilitation and a greater inhibition effect as a function of context.

These results therefore suggest one of two interpretations: either the blocked presentation slows down decisions following sentence contexts without affecting decisions following neutral contexts, or it speeds up decisions following a neutral context without affecting decisions following sentence contexts.

When the decision latencies are compared directly between Experiments 1 and 2, the results are more consistent with the first possibility: the decision latencies in the neutral context conditions are relatively invariant across the two experiments (see Tables 2 and 3). However, it should be noted that the two experiments involved two different groups of subjects, and it is possible that one group
Table 4

Mean Decision Latencies (in Milliseconds) for the 19 Fastest Subjects in Experiments 1 and 2

\begin{tabular}{llll}
\hline $\begin{array}{l}\text { Experi- } \\
\text { ment 1 }\end{array}$ & $\begin{array}{c}\text { Experi- } \\
\text { ment 2 }\end{array}$ & $\begin{array}{c}\text { Differ- } \\
\text { ence }\end{array}$ \\
\hline
\end{tabular}

Sentence context

\begin{tabular}{lllc} 
Word & & & \\
High frequency & 614 & 615.5 & -1.5 \\
Low frequency & 672.5 & 657 & 15.5 \\
Nonword & 783 & 774 & 9 \\
$M$ & 713.2 & 705.2 & 8 \\
\hline
\end{tabular}

Neutral context

Word

\begin{tabular}{clll} 
High frequency & 628 & 597 & 31 \\
Low frequency & 670 & 629 & 41 \\
Nonword & 815 & 766 & 49 \\
$M$ & 732 & 689.5 & 42.5 \\
\hline
\end{tabular}

involved subjects who were slower overall than the other group. This fluctuation in absolute reaction time appears to have occurred in Experiments 1 and 2, because in Experiment 1 , there was only 1 subject whose average decision latency for word targets exceeded 900 $\mathrm{ms}$, whereas in Experiment 2, there were 5 such subjects. Therefore, there is a possibility that these slow subjects in Experiment 2 (where a blocked presentation was used and therefore a faster neutral decision latency was expected) may have masked the shift in the absolute decision latency for the neutral context conditions. To check this possibility, in the following analysis, the 5 slowest subjects were eliminated from Experiments 1 and 2. This procedure yielded the following mean decision latencies (see Table 4).

As can be seen from Table 4, whereas the decision latencies for the sentence context conditions were relatively stable across experiments (averaged over word and nonword decisions, $713.2 \mathrm{~ms}$ in Experiment 1 and $705.2 \mathrm{~ms}$ in Experiment 2), the decision latencies for the neutral context conditions were substantially faster in Experiment 2 (732 $\mathrm{ms}$ in Experiment 1 and $689.5 \mathrm{~ms}$ in Experiment 2). This observation was supported by the significant contrast testing of the interaction between Experiment (Experiment 1 vs. 2) and Context (sentence vs. neutral context conditions), which yielded 
the following $F$ values: $F_{1}(1,36)=9.111$, $M S_{\mathrm{e}}=627.6 ; \quad F_{2}(1,141)=8.368, \quad M S_{\mathrm{e}}=$ 4,928.553. This pattern of data therefore provides support for the interpretation that the overestimate of baseline decision latency has its origin in the mixed presentation of sentence and neutral context conditions.

A large fluctuation in absolute reaction times between different subjects are not uncommon; for example, in a series of experiments conducted by Stanovich and West (1983, Experiments 6, 7, \& 8), the mean naming latencies for the same targets varied by more than $100 \mathrm{~ms}$, despite the fact that subjects were from the same population. Such a between-groups difference in overall response latency, of course, could lead to a high Type II error rate.

For this reason, in the present analysis, rather than directly comparing decision latencies between experiments, the pattern of facilitation and inhibition (which is assessed within-subjects) was examined for each experiment separately. When this was done, the facilitation and inhibition profile obtained favored the second interpretation just discussed, namely, that the blocked presentation acted to speed up decisions following a neutral context while keeping decisions following sentence contexts unaffected.

If it is accepted that different modes of presentation result in a shift in the decision latency following a neutral context, what is the underlying mechanism for this shift? An answer to this question may be found by examining the strategies available for responding to the targets in the neutral context condition in the two modes of presentation.

In a lexical decision task where the target is always presented in isolation, it is generally assumed that the lexical entry is accessed on the basis of its physical (orthographic) features. It may be further assumed in the present experiments that nonword targets also access lexical entries, because nonword targets in the present and also some previous experiments were generated by substituting a letter of a word (e.g., the nonword yeir is generated from year) so that they were sufficiently similar to an existing word to activate its entry in lexical memory (cf. Chambers, 1979). This suggests that a subsequent post-access check may be necessary before a lexical decision can be made. That is, word and nonword targets are not automatically discriminated by simply monitoring whether or not a lexical entry was accessed, but rather depend on a detailed comparison between the accessed entry and the actual target being carried out. When a target is presented without context, obviously only the physical features of the accessed entry can be used for this comparison. Therefore, with blocked presentation of neutral context condition, this is the process most likely to be used.

On the other hand, when the target is presented following a sentence context, the meaning of an accessed entry may be used in addition to its physical features during post-access checking. Specifically, subjects can be certain that the target is a word if the sentence as a whole makes sense. At the same time, sentential meaningfulness cannot be used as the sole basis for making lexical discriminations, because of the possible presence of incongruous word targets. Therefore, subjects may have adopted the following twostage decision-making process, similar to the one suggested by Rips, Shoben, and Smith (1973) for the category verification task, and more recently, by Balota and Chumbley (1984) for the lexical decision task. During the first stage, sentential meaningfulness as well as the physical features of an accessed entry are examined, and if the combined evidence in favor of the entry exceeds a certain higher criterion, or falls below a lower criterion, a decision is made rapidly. Thus for congruous word targets and for incongruous nonword targets (which would always occur when a nonword follows a sentence context), a rapid word or nonword decision can be made. However, for targets whose evidence falls in the intermediate range between the two cutoff points, further processing must be carried out before a decision can be reached. During this second stage, the physical features of the accessed entry are scrutinized in order to avoid responding incorrectly to incongruous word targets. As there would be no information concerning sentential meaningfulness for targets following a neutral context, decisions regarding these targets are also likely to be deferred until the second stage. Thus compared with blocked presentation, 
mixed presentation results in longer decision latencies for targets following a neutral context because of the hypothesized extra processing stage involved in the latter case.

According to this account, which we will refer to as the two-stage decision strategy, the faster decision latency for words following congruous sentence contexts relative to the neutral contexts in the Experiment 1 is explained in terms of the difference between the time to complete only the first stage of processing and the time to complete both stages of processing. However, in Experiment 2 , where a blocked presentation was used, this facilitation effect was statistically unreliable. This may be because the first stage of the two-stage decision process takes effectively as long to complete as the single-stage decision process used for targets presented in blocked neutral trials and is based only on physical features of the target. Similarly, the nonword facilitation effect obtained in Experiment 1 can be explained in terms of whether a decision is made on the basis of first-stage processing or second-stage processing. The absence of any nonword facilitation in Experiment 2 can again be explained in terms of the equivalent time taken to complete the first stage of the two-stage decision process on the one hand and the single-stage decision process on the other.

It should be pointed out that this explanation so far presented is still not sufficient to account for the inhibition effect obtained in Experiment 1. That is, if a decision for a target word following a neutral context and a decision following an incongruous sentence context must both await the completion of the second-decision stage, there should have been no difference in the decision latencies following these two types of target words, which was not the case. Thus the effect of sentence context cannot be explained solely in terms of a two-stage decision strategy based only on sentential meaningfulness. A possible locus of this inhibition effect will be discussed later.

It should be further noted that unlike the models of sentence context effects mentioned earlier, the account advanced here views sentence contexts as essentially affecting processes that take place after lexical access. Consequently, it is quite consistent with the absence of an interaction between the effects of frequency and facilitation in both Experiments 1 and 2. That is, because the process of lexical access itself is unaffected by sentence context, there is no reason to expect a reduced effect of frequency for words following congruous sentence contexts relative to the neutral context condition. ${ }^{3}$ Similarly, the absence of a correlation between Cloze probability and the size of facilitation observed in the two experiments is consistent with the present account, because sentence contexts are not assumed to be used to predict likely target words.

This two-stage decision strategy model can also provide an explanation for previous findings that have so far lacked an adequate explanation. For example, it may be recalled that the finding that nonword responses are slower following a neutral context consisting of the words They said it was the relative to a sentence context condition (West \& Stanovich, 1982) was incompatible with any of the explanations of the nonword facilitation effect mentioned earlier. With an added assumption, however, the present account can accommodate this finding. The addition is to suggest that a sentence consisting of the above type of neutral context and a target word (e.g., They said it was the tooth) is neither semantically incongruous, nor highly meaningful. If this is the case, then, targets following this type of context must also await the completion of the second stage of decision process before a decision can be reached. In other words, just as a neutral context consisting of a row of $X$ s produces an overestimate of the baseline decision latency with a mixed presentation, the neutral context con-

\footnotetext{
${ }^{3}$ The present interpretation also predicts additive effects of frequency and inhibition. In all three experiments reported in this article, there was a nonsignificant trend toward a greater effect of frequency for words following an incongruous sentence context compared with a neutral context. If this effect is genuine, it does compromise the present view that the effects of frequency and context do not interact. However, given the fact that the interaction never reached statistical significance either on subjects or items analyses, and given also the absence of such a finding in other experiments using sentence contexts (e.g., Forster, 1981; Schuberth \& Eimas, 1977; Stanovich \& West, 1981, 1983; West \& Stanovich, 1982), it is considered to be an unreliable effect.
} 
sisting of the words $I t$ was the also produces an overestimate.

The present model is also able to account for Fischler and Bloom's $(1979,1980)$ finding of equivalent decision latencies for a neutral context consisting of a row of $X \mathrm{~s}$ and that consisting of sentence fragments, which, when combined with the target, were always semantically incongruous. In Fischler and Bloom's experiments, these neutral trials were blocked and were presented to a group of subjects different from the group of subjects presented with the congruous and incongruous sentence context conditions. Because sentential meaningfulness necessarily cannot be used to discriminate between word and nonword targets following either type of neutral contexts presented, there would have been no basis for adopting a two-stage decision process. Hence, these two types of neutral context conditions produced equivalent baseline decision latencies.

Thus, the proposed two-stage decision model can handle most of the findings obtained in Experiments 1 and 2 of this article and in other experiments reported previously. However, one finding that at first glance appears to be inconsistent with this account is that of Forster's (1981) where no facilitation was obtained for word or nonword decisions. Because a mixed presentation was used in Forster's experiment, it might have been expected that targets following the neutral context should have been responded to on the basis of two-stage processing. In Forster's experiment, the neutral context consisted of a random list of words (e.g., As its awful her fun doing were has under), and a rapid serial visual presentation (RSVP) was used to display these words. It should be pointed out that because Sanocki and Oden (1984) also used a random list of words as a neutral context and did obtain a significant nonword facilitation effect, the absence of the effect in Forster's experiment cannot be accounted for solely in terms of the type of neutral context.

One way to resolve the discrepancy between the present explanation for nonword facilitation and Forster's finding is to assume that subjects in Forster's experiment did not use sentential meaningfulness as a basis for making word-nonword discriminations and therefore did not (need to) adopt a two-stage decision process, as we would otherwise expect. There are some reasons for proposing this possibility. First, as Forster himself has pointed out, a random list of words cannot be integrated semantically or syntactically with a target word: at the same time, unlike a row of $X s$, a random list of words may be difficult to distinguish from a sentence context. ${ }^{4}$ Second, this difficulty may have been compounded by the use of RSVP to display the words of context. That is, it would have been difficult to discern whether the sentence as a whole did not make sense because the target was incongruous with the context, or because the context itself could not be comprehended. Consequently, subjects may have been discouraged from using sentential meaningfulness as a basis for making lexical decisions, because such a strategy would result in a wrong nonword decision for word targets following a random list of words, as well as those following an incongruous sentence context. This means that there would not have been any incentive to adopt a two-stage decision process. Thus it follows that there were no facilitation effects for word or nonword decisions.

In contrast, in Sanocki and Oden's (1984) experiment, RSVP was not used, but rather, the words of context were presented at once and the duration of the context display was under the subjects' control. Consequently, subjects would have been able to decide whether or not the context itself made sensein fact, subjects were required to make a "sensibleness judgment" on each contextand therefore able to exploit sentential meaningfulness to make word-nonword discriminations.

What is being argued here is that Forster's (1981) finding can be accommodated within

\footnotetext{
${ }^{4}$ It could be argued that because the row of $X$ s used as the neutral context in Experiments 1 and 2 was readily distinguishable from a sentence context, subjects should have been able to switch their decision strategy following different types of context. The effect of blocked versus mixed presentation of context conditions observed here suggests this is precisely what subjects do not do during a mixed series of trials. Other findings of the effects of blocking/mixing different items (e.g., Kiger \& Glass, 1981) support the contention that once a subject adopts a particular decision strategy, it is difficult to modify it within a given block of trials.
} 
the present account if it is assumed that subjects modify their decision strategy depending on how useful sentential meaningfulness is in making word-nonword discrimination. The final experiment tested this assumption. In this experiment, as in Experiment 1 , a mixed presentation was used, with the following changes. First, the incongruous sentence contexts used in Experiment 1 were replaced by a new type of sentence context. These were sentences that were clearly semantically anomalous even before a target word completed the sentence (e.g., The sparrow mended the road in the SOFA). With the incongruous sentence contexts used in Experiment 1 , it is not clear whether the sentence as a whole may be meaningful until a target is presented. Therefore subjects could not know beforehand whether a decision strategy based on sentential meaningfulness would be costly. On the other hand, with the anomalous sentence contexts it is obvious that no word can make the sentence as a whole meaningful. Thus the introduction of this type of sentence context was expected to alert the subjects to the fact that a decision strategy based on sentential meaningfulness would not be useful.

A second difference between Experiments 1 and 3 was the type of nonword targets. In Experiment 1, the nonword targets were not physically similar to any word that may have been a meaningful completion for the preceding sentence context. In Experiment 3, the nonword targets following congruous sentence contexts were generated by substituting a letter of a word that was semantically congruous with the context [e.g., The cows were taken to the DAIKY (DAIRY)]. These nonwords were assumed to access lexical entries corresponding to the original word, and thus a decision strategy based on sentential meaningfulness would lead to an incorrect word decision. These two factors ${ }^{5}$ were designed, therefore, to discourage subjects from using sentential meaningfulness as a basis for making lexical decisions. Consequently it was expected that in Experiment 3, as in Forster's (1981) experiment, there would be no facilitation of word or nonword decisions following congruous sentence contexts relative to the neutral context condition.

\section{Experiment 3}

\section{Method}

Subjects. Twenty-four additional subjects from the same population as used in previous experiments participated in this experiment.

Stimuli. There were 72 word targets in this experiment assigned to the six conditions resulting from the $2 \times 3$ combinations of frequency (high and low) and context (congruous, anomalous, and neutral). The high-frequency targets had 100 or more occurrences per million (average 183.4 per million) and the low-frequency items, 20 or less per million (average 8.6 per million) according to the Kucera and Francis (1967) word frequency count.

The congruous sentence contexts were generated by using the same general procedure as in Experiment I, with the constraint that the target word was not highly predictable from the context (Cloze probability $<0.8$ ). The anomalous sentences were generated by changing some words of the congruous sentence with the following constraints: (a) that the number of words in each sentence matched that of the congruous sentence and (b) that the sentence was semantically anomalous but syntactically well-formed. The neutral context consisted of the same row of $X \mathbf{s}$ used in previous experiments. Examples of contexts and targets are displayed in Table 5.

There were also 72 nonword targets presented under the same three context conditions. Additional 72 congruous sentences were generated by the same procedure described earlier. Each nonword target was then generated by changing one letter of the final word in the sentence, for example, the nonword daiky was generated from the word dairy in the sentence, The cows were taken to the DAIRY. The anomalous sentence contexts and the neutral contexts were also generated by the same procedure used for word targets.

Procedure. The apparatus, timing characteristics and the instructions given to the subjects were identical to those in Experiment 1.

\section{Results and Discussion}

The analysis of Experiment 3 was essentially the same as that in Experiment 1. The mean latency for correct responses and error rates for word decisions are displayed in Table 6.

For word decisions, the latency data showed that the main effect of frequency was significant, $F_{1}(1,23)=65.203, M S_{\mathrm{e}}=4,884.147$

\footnotetext{
${ }^{5}$ Any absence of nonword facilitation in Experiment 3 arguably may be attributed to either one of these factors, namely, the presence of anomalous sentence contexts, or the inclusion of nonword targets that are orthographically similar to an existing word. In a pilot test manipulating only one of these factors, the results were equivocal, suggesting that both factors were necessary to discourage subjects from using sentential meaningfulness as a basis for making lexical decision.
} 
Table 5

Examples of Targets and Contexts Used and the Number of Trials Per Condition in Experiment 3

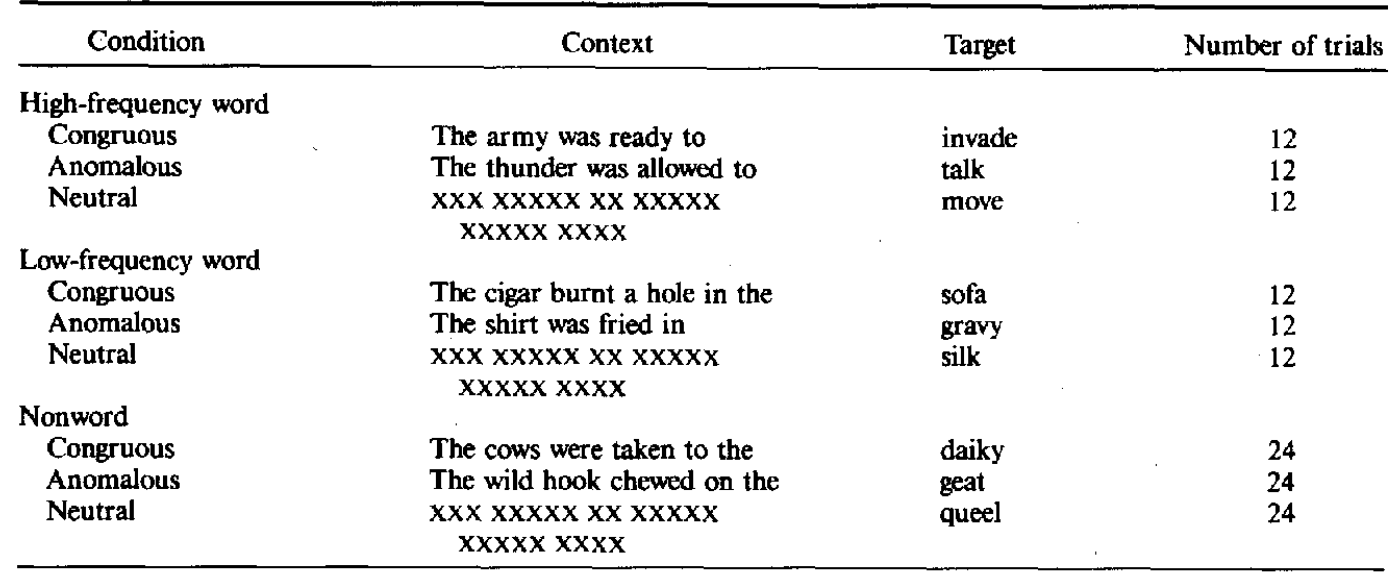

and $F_{2}(1,70)=31.712, M S_{\mathrm{e}}=9,666.192$. Overall, the high-frequency words were responded to $94.3 \mathrm{~ms}$ faster than low-frequency words. As can be seen from the table, the facilitation effect was negligible and nonsignificant, $F_{1}(1,23)<1.0$ and $F_{2}(1,70)<1.0$. The 42-ms inhibition effect, on the other hand, was significant, $F_{1}(1,23)=10.519$, $M S_{\mathrm{e}}=4,065.975$ and $F_{2}(1,70)=5.555$, $M S_{\mathrm{e}}=7,430.23$. None of the interactions involving these contrasts were significant. $[F<$ 1.0 in all cases except for the inhibition by frequency interaction on subjects analysis: $\left.F_{1}(1,23)=1.855, M S_{\mathrm{e}}=4,396.226.\right]$

The error rate data also showed a significant main effect of frequency, $F_{1}(1,23)=30.25$, $M S_{\mathrm{e}}=0.444$ and $F_{2}(1,70)=10.591, M S_{\mathrm{e}}=$ 0.846 . The facilitation effect did not reach significance, $F_{1}(1,23)=2.853, M S_{\mathrm{e}}=0.716$ and $F_{2}(1,70)=3.708, M S_{\mathrm{e}}=0.316$. Contrary to the pattern of the latency data, fewer errors were made in the anomalous sentence context condition than in the neutral context condition, however, this trend was not reliable, $F_{1}(1,23)=1.117, M S_{\mathrm{e}}=0.933$ and $F_{2}(1$, 70) $=1.583, M S_{\mathrm{e}}=0.355$. The facilitation by frequency interaction approached, but did not reach, significance, $F_{1}(1,23)=3.833$, $M S_{\mathrm{e}}=0.391$ and $F_{2}(1,70)=3.708, M S_{\mathrm{e}}=$ 0.316 . The inhibition by frequency interaction was also nonsignificant, $F_{1}(1,23)<1.0$ and $F_{2}(1,70)<1.0$.

The nonword decision data are also displayed in Table 6. For the nonword data, two contrasts were tested: facilitation (comparing decisions in the congruous sentence context condition with the neutral context condition) and inhibition (comparing the decisions in the anomalous sentence context condition with the neutral context condition). For the latency data, neither contrasts reached significance: facilitation, $F_{1}(1,23)=1.016, M S_{\mathrm{e}}=$ $6,308.808$ and $F_{2}(1,71)<1.0$; inhibition, $F_{1}(1,23)<1.0$ and $F_{2}(1,71)<1.0$. These contrasts were also nonsignificant for the error data as well ( $F<1.0$ in all cases).

The results of Experiment 3 thus indicated

Table 6

Mean Latencies (in Milliseconds) and Percentage Error Rates (\% E) for Word and Nonword Decisions in Experiment 3

\begin{tabular}{lllllll}
\hline & \multicolumn{6}{c}{ Frequency } \\
\cline { 2 - 7 } Condition & High & \% E & Low & \% E & $\begin{array}{c}\text { Differ- } \\
\text { ence }\end{array}$ & \% E \\
\hline Word & & & & & & \\
congruous & 652 & 1.0 & 741 & 3.8 & 89 & 2.8 \\
$\begin{array}{l}\text { anomalous } \\
\text { neutral }\end{array}$ & 684 & 0.4 & 800 & 5.9 & 116 & 5.5 \\
$\begin{array}{l}\text { Nonword } \\
\text { congruous }\end{array}$ & 854 & 1.4 & 739 & 8.3 & 78 & 6.9 \\
anomalous & 856 & 4.7 & & & & \\
neutral & 844 & 5.2 & & & & \\
\hline
\end{tabular}

Note. Facilitation and inhibition effects for word contrasts were $1.8(\% \mathrm{E}=2.5)$ and $42(\% \mathrm{E}=-1.7)$, respectively. Facilitation and inhibition effects for nonword contrast were $-10(\% \mathrm{E}=0.5)$ and $12(\% \mathrm{E}=-0.2)$, respectively. 
that word decisions following congruous contexts were not facilitated relative to the neutral context condition, although significant inhibition was obtained for word decisions following anomalous sentence contexts. On the other hand, context conditions had no effect on nonword decisions. The absence of facilitation for both word and nonword targets suggests that sentential meaningfulness was not used to assist the word-nonword discrimination process and therefore supports the prediction made at the outset of the experiment. That is, under conditions where sentential meaningfulness cannot be used effectively for the majority of trials, subjects abandon a decision strategy based on this factor.

There are also two additional findings that further lend support for this view. First, unlike the two previous experiments, no inhibition effect was obtained for the error rate measure for word targets. It may be noted that Forster (1981, Experiment 3) also obtained an inhibition effect only with the decision latency measure and not with the error rate measure. This finding indicates that subjects were not biased towards responding nonword when the sentence as a whole was meaningless. This absence of an inhibition effect on the error rate was not offset by a greater inhibition effect on the decision latency measure either. On the contrary, the size of the inhibition effect is, if anything, smaller in this experiment $(42 \mathrm{~ms})$ than in the previous experiments (44.5 $\mathrm{ms}$ and $81.5 \mathrm{~ms})$. Second, the overall effect of context on word decisions $\left[R T_{\text {incongruous }}-\mathrm{RT}_{\text {congruous }}\right]$ was smaller in Experiment 3 than in previous experiments. This finding is also consistent with the idea that subjects did not use sentential meaningfulness to bias their decisions.

These findings therefore provide support for the interpretation of Forster's (1981) finding put forward earlier; namely, no nonword facilitation was obtained in his experiment because subjects' realization that sentential meaningfulness was not a useful factor under these conditions in making lexical discriminations led them to abandon the two-stage decision strategy.

Although the absence of facilitation effects was expected, the effect of anomalous sentence contexts on word decisions has yet to be explained. The nature of this inhibition effect will be discussed further in the following section.

\section{General Discussion}

The aim of this article has been to investigate the locus of nonword facilitation effects that have been obtained in lexical decision tasks. The results of Experiments 1 and 2 indicated that nonword facilitation is eliminated when the neutral context condition is presented in a separate block of trials. This finding was interpreted in terms of whether or not targets following a neutral context are affected by a two-stage decision strategy that involves sentential meaningfulness as one of the decision criteria.

The results of Experiment 3 indicated that when sentential meaningfulness cannot be used to discriminate between word and nonword targets in a majority of trials, no facilitation effect is obtained either for word or nonword targets even when a mixed presentation of sentence context and neutral context conditions is used. In summary, the results of the three experiments suggest that nonword facilitation arises from an overestimate of baseline decision latency which has its origin in an optional decision strategy based on sentential meaningfulness.

This interpretation of the results still leaves one question open: What is the source of the inhibition effects obtained in Experiments 1 and 3? In Experiment 1, if both targets following a neutral context and an incongruous sentence context must await the completion of the second decision stage, why did they not produce equal decision latencies? In Experiment 3, if a decision strategy based on sentential meaningfulness was not used, what mechanism could be responsible for the inhibition effect? These results suggest that unlike the facilitation effect, the inhibition effect has its source outside the decision strategy described thus far.

The explanation that best fits the present evidence of inhibition effects is one that has been proposed by Forster (1981). As we have also assumed, he viewed lexical access as an autonomous subprocess that is driven solely by the physical features of a word and is unaffected by previously occurring semantic information. His view of language processing 
suggests that it is a linear hierarchy of operations, involving subprocesses such as lexical access, syntactic analysis, and semantic processing. Each subprocess is considered to be autonomous and accepts its input only from the next lowest level and from no other source. The output of these subprocesses is then communicated to a general problem solver (GPS), which is responsible for integrating these pieces of information as well as governing any (conscious) decisions concerning them. Within this framework, the inhibitory effect of sentence contexts is thought to have its source in the integration process. When the meaning of an entry (that has been accessed on the basis of the word's physical features) cannot be integrated with the preceding context to make the sentence as a whole meaningful, a search is initiated to find an alternative entry. If no entry can make the sentence meaningful, then one of the previously rejected entries must be reinstated, thus resulting in a slower response in the case of targets following an incongruous/ anomalous context relative to a neutral context.

This interpretation of the inhibition effect is appealing for a number of reasons. First, because it places the locus of the effect of sentence context after lexical access, which is consistent with the present account of nonword facilitation. Second, it is compatible with the earlier finding in Experiments 1 and 2 that the effects of frequency and context are additive rather than interactive. Third, and most important, this interpretation can account for the effect in Experiment 3 of anomalous sentence contexts when sentential meaningfulness is not used to bias lexical decisions. That is, because subjects in that experiment were not biased towards responding nonword when the sentence as a whole did not make sense, there was no inhibition effect on the error rate data. At the same time, an inhibition effect on the latency data was obtained because of the failure of the word target to be integrated with the anomalous context to make a meaningful sentence.

There is, however, one potential problem with this account. It has been assumed that because the nonword targets used in the present set of experiments were similar to existing words, they also activated lexical entries. If, as we have suggested, target words are responded to more slowly following an anomalous sentence context, why wasn't there an inhibition effect for nonword decisions as well? An answer to this question may be found by considering how a nonword decision is made. For such a decision to occur, it must be established that there are no existing lexical entries that match the presented target. That is, unlike a word decision, which is made when a match is found between an entry and a target, a nonword decision must necessarily involve an exhaustive check (and subsequent rejection) of all entries that are sufficiently similar to the target. In the case of a word decision, the inhibition effect following an anomalous sentence context was proposed earlier to be the result of a premature rejection of an entry or entries, which led to an exhaustive check of all other activated entries before the original entry was reinstated. For nonword decisions, however, no reinstatement of an entry is warranted, so in this case then there should be minimal effect of anomalous sentence contexts because an exhaustive check of activated entries is involved regardless.

We conclude this article by discussing some of the implications of the present view. First, it suggests that in experiments using a lexical decision task, there are two sources of sentence context effects: a two-stage decision strategy based on sentential meaningfulness that affects both word and nonword targets and a post-access integration process leading to inhibition effects specific to word targets. If we assume that the former is an artifact of the peculiar type of decision required in the lexical decision task, then it would seem to follow that in the majority of previous experiments using this task, the effect of sentence context, in particular, the facilitation effect, has been overestimated. What we have shown is that while a blocked presentation of sentence and neutral context conditions reduces the likelihood that decisions for targets following a neutral context will be disadvantaged by this decision strategy, it does not preclude its occurrence for targets following sentence contexts. $^{6}$

\footnotetext{
${ }^{6}$ Recently Jonides and Mack (1984) warned against the use of blocked presentation and instead recommended
} 
Second, there is some indication that the proposed two-stage decision strategy may also be involved in experiments involving singleword contexts. For example, de Groot (1983, 1984) has recently put forward the view that the effects of single-word contexts are largely attributable to a "post-access coherence check," which involves a search of the semantic relation between the target and the context word. If there is a compatible relation, the subject will tend to respond word; if not, there will be a bias towards responding nonword. This post-access coherence check would seem to be very similar to the decision strategy based on sentential meaningfulness described in this article. It would be interesting to see whether, in the absence of this decision strategy, the effect of a single-word context is also inhibitory, as we have shown here with sentence contexts.

Finally, a comment is in order regarding the role of post-access inhibition in reading. It has been claimed by West and Stanovich (1982) that post-access inhibition is also an experimental artifact of the lexical decision task, which requires an "ecologically unprecedented situation of making a yes/no response to linguistic material" (p. 396). We would argue, however, against this view. We suggest that post-access inhibition itself may serve a useful function, as Fischler and Bloom (1980) have suggested, "as a signal that perception

the mixed presentation. However, it should be pointed out that they did not present any a priori reason for favoring the latter, apart from that based on a finding by Jonides (1980, Experiment 2). Jonides reported that a separate presentation of a neutral condition yielded reaction times that were slower than the corresponding reaction times for a mixed presentation condition. He therefore concluded that subjects in the separate presentation condition were performing suboptimally. However, it should be noted that different groups of subjects were involved in the two presentation conditions. That is, this finding may have been due to individual differences in absolute reaction times, rather than an effect of presentation condition per se, as has been implicitly assumed.

In contrast, we favor a blocked presentation, because it provides a better estimate of the baseline decision latency for targets following a neutral context that is unaffected by a decision strategy based on sentential meaningfulness. It may also be added that because it precludes this decision strategy (for targets following a neutral context), this mode of presentation yields a faster estimate of the baseline. or comprehension has failed and that some reanalysis is called for" (p. 224).

At least two situations come to mind in which such a signal may be useful. The first of these is when a sentence contains a lexically ambiguous word (e.g., organ, box). Recent experimental evidence suggests that sentence contexts do not guide lexical access to the semantically appropriate meaning of a homograph, but that multiple meanings are activated momentarily even in the presence of a given context (e.g., Onifer \& Swinney, 1981; Seidenberg, Tanenhaus, Leiman, \& Bienkowski, 1982; Tanenhaus, Leiman, \& Seidenberg, 1979). What the sentence contexts do in this situation is instead to inhibit the semantically inappropriate meaning(s) after they have been accessed. A second situation in which post-access inhibition may be useful is in reading a word that is physically similar to another word. For example, Forster (1978) reported his experience of reading the sentence, "Sherlock Holmes was capable of almost superhuman excretions." Noting the gross implausibility of the sentence, he reexamined the sentence to discover that it actually contained the word exertions. In this case, the failure of the sentence to make sense alerted the reader to the occurrence of an error in the perception of a word. The fact that these two situations can occur often in normal reading leads one to suggest that post-access inhibition is not simply an artifact, but may play an important role in the process of word recognition that warrants further investigation.

\section{References}

Antos, S. J. (1979). Processing facilitation in a lexical decision task. Journal of Experimental Psychology: Human Perception and Performance, 5, 527-545.

Balota, D. A., \& Chumbley, J. I. (1984). Are lexical decisions good measure of lexical access? The role of word frequency in the neglected decision stage. Journal of Experimental Psychology: Human Perception and Performance, 10, 340-357.

Becker, C. A. (1979). Semantic context and word frequency effects in visual word recognition. Journal of Experimental Psychology: Human Perception and Performance, 5, 252-259.

Becker, C. A. (1980). Semantic context effects in visual word recognition: An analysis of semantic strategies. Memory \& Cognition, 8, 498-512.

Bloom, P. A., \& Fischler, I. (1980). Completion norms for 329 sentence contexts. Memory \& Cognition, 8, $631-642$. 
Chambers, S. (1979). Letter and order information in lexical access. Journal of Verbal Learning and Verbal Behavior, 18, 225-241.

de Groot, A. M. B. (1983). The range of automatic spreading activation in word priming. Joumal of Verbal Learning and Verbal Behavior, 22, 417-436.

de Groot, A. M. B. (1984). Primed lexical decision: Combined effects of the proportion of related primetarget pairs and the stimulus-onset asynchrony. Quarterly Journal of Experimental Psychology, 36A, 253280.

Fischler, I., \& Bloom, P. A. (1979). Automatic and attentional processes in the effects of sentence contexts on word recognition. Journal of Verbal Learning and Verbal Behavior, 18, 1-20.

Fischler, I., \& Bloom, P. A. (1980). Rapid processing of the meaning of sentences. Memory \& Cognition. 8 , 216-225.

Forster, K. I. (1976). Accessing the mental lexicon. In R. J. Wales \& E. Walker (Eds.), New approaches to language mechanisms. Amsterdam: North-Holland.

Forster, K. I. (1978). Lexical and sentential context effects in word recognition. Paper presented at the Fifth Australian Experimental Psychology Conference, La Trobe University, Victoria, Australia.

Forster, K. I. (1981). Priming and the effects of sentence and lexical contexts on naming time: Evidence for autonomous lexical processing. Quarterly Journal of Experimental Psychology, 33A, 465-496.

Forster, K. I., \& Chambers, S. M. (1973). Lexical access and naming time. Journal of Verbal Learning and Verbal Behavior, 12, 627-635.

Glanzer, M., \& Ehrenreich, S. L. (1979). Structure and search of the internal lexicon. Journal of Verbal Learning and Verbal Behavior, 18, 381-398.

Jonides, J. (1980). Towards a model of the mind's eye's movement. Canadian Journal of Psychology, 34, 103112.

Jonides, J., \& Mack, R. (1984). On the cost of benefit of cost and benefit. Psychological Bulletin, 96, 29-44.

Kiger, J. I., \& Glass, A. L. (1981). Context effects in sentence verification. Journal of Experimental Psychology: Human Perception and Performance, 7. 688700 .

Kleiman, G. M. (1980). Sentence frame contexts and lexical decisions: Sentence acceptability and wordrelatedness effects. Memory \& Cognition, 8, 336-344.

Kucera, H., \& Francis, W. N. (1967). Computational analysis of present-day American English. Providence, RI: Brown University Press.

Morton, J. (1969). Interaction of information in word recognition. Psychological Review, 76, 165-178.

Neely, J. H. (1976). Semantic priming and retrieval from lexical memory: Evidence for facilitatory and inhibitory processes. Memory \& Cognition, 5, 648-654.

Neely, J. H. (1977). Semantic priming and retrieval from lexical memory: Roles of inhibitionless spreading activation and limited-capacity attention. Journal of $E x$ perimental Psychology: General, 106, 226-254.

Onifer, W., \& Swinney, D. A. (1981). Accessing lexical ambiguities during sentence comprehension: Effects of frequency of meaning and contextual bias. Memory \& Cognition, 9, 225-236.

Rips, L. J., Shoben, E. J., \& Smith, E. E. (1973). Semantic distance and the verification of semantic relations. Journal of Verbal Learning and Verbal Behavior, 12, 1-20.

Rosch, E. (1975). Cognitive representations of semantic categories. Journal of Experimental Psychology: General, 104, 192-233.

Sanford, A. J., Garrod, S., \& Boyle, J. M. (1977). An independence of mechanism in the origins of reading and classification-related semantic distance effects. Memory \& Cognition, 5, 214-220.

Sanocki, T., \& Oden, G. C. (1984). Contextual validity and the effects of low-constraint sentence contexts on lexical decisions. Quarterly Journal of Experimental Psychology, 36A, 145-156.

Scarborough, D. E., Gerard, L., \& Cortese, C. (1977). Frequency and repetition effects in lexical memory. Journal of Experimental Psychology: Human Perception and Performance, 3, 1-17.

Schuberth, R. E., \& Eimas, P. D. (1977). Effects of context on the classification of words and nonwords. Journal of Experimental Psychology: Human Perception and Performance, 3, 27-36.

Schuberth, R. E., Spoehr, K. T., \& Lane, D. M. (1981). Effects of stimulus and contextual information on the lexical decision task. Memory \& Cognition, 9, 68-77.

Seidenberg, M. S., Tanenhaus, M. K., Leiman, J. M., \& Bienkowski, M. (1982). Automatic access of the meanings of ambiguous words in context: Some limitations of knowledge-based processing. Cognitive Psychology, 14, 489-537.

Stanovich, K. E., \& West, R. F. (1979). Mechanisms of sentence context effects in reading: Automatic activation and conscious attention. Memory \& Cognition, 7, 77-85.

Stanovich, K. E., \& West, R. F. (1981). The effect of sentence context on ongoing word recognition: Tests of a two-process theory. Journal of Experimental Psychology: Human Perception and Performance, 7, 638678.

Stanovich, K. E., \& West, R. F. (1983). On priming by a sentence context. Joumal of Experimental Psychology: General, 112, 1-36.

Tanenhaus, M. K., Leiman, J. M., \& Seidenberg, M. S. (1979). Evidence for multiple stages in the processing of ambiguous words in syntactic contexts. Journal of Verbal Learning and Verbal Behavior, 18, 427-440.

West, R. F., \& Stanovich, K. E. (1982). Source of inhibition in experiments on the effect of sentence context on word recognition. Journal of Experimental Psychology: Learning, Memory, and Cognition, 8, 385399.

Whaley, C. P. (1978). Word-nonword classification time. Journal of Verbal Learning and Verbal Behavior, 17. 143-154.

Received July 1982

Revision received October 31, 1984 